\title{
Utilização da Programação Linear na Otimização de Resultados de uma Empresa do Ramo de Transporte Rodoviário de Cargas
}

\begin{abstract}
Roberto Carlos Klann Mestrado em Ciências Contábeis pela Universidade Regional de Blumenau - FURB Rua Dorval Luz, 147. Brusque/SC. CEP: 88352-400 E-mail:rklann@al.furb.br

Paulo Roberto da Cunha Doutorado em andamento em Ciências Contábeis e Administração pela Universidade Regional de Blumenau - FURB Rua Antônio da Veiga, 140. Victor Konder. Sala D 202. Blumenau/SC. CEP: 89012-900

E-mail: pauloccsa@furb.br

Júlio Orestes da Silva Mestrado em andamento em Ciências Contábeis pela Universidade Regional de Blumenau - FURB Rua Antônio da Veiga, 140. Victor Konder. Sala D 202. Blumenau/SC. CEP: 89012-900 E-mail: juliosilva@al.furb.br

Jorge Eduardo Scarpin Doutorado em Ciências Contábeis pela Universidade Regional de BlumenauFURB, Professor da FURB, SC Rua Antônio da Veiga, 140. Victor Konder. Sala D 202. Blumenau/SC. CEP: 89012-900 E-mail: jorgescarpin@furb.br
\end{abstract}

\section{RESUMO}

Trata o presente artigo de um estudo sobre a otimização de resultado de uma empresa de transporte rodoviário de cargas, que apresenta capacidade de carga ociosa. Essa empresa faz parte de um setor altamente competitivo no Brasil, que necessita de ferramentas gerenciais que possam auxiliar seus gestores no processo de tomada de decisão. O objetivo do artigo é aplicar a programação linear para otimizar o resultado dessa empresa, estabelecida na Região do Vale do Itajaí, Estado de Santa Catarina. O estudo é de caráter exploratório com abordagem quantitativa, e os dados foram obtidos por meio de pesquisa documental, com consulta a documentos primários e secundários da empresa. Os resultados apontam que a empresa necessita incrementar o volume transportado por sua matriz e pelas duas filiais estabelecidas no Estado de Santa Catarina. Dadas as restrições na demanda de cada filial, bem como à restrição de veículos possuídos pela empresa para esse serviço, a matriz da empresa deve aumentar o seu volume transportado em aproximadamente 17\%, a Filial SC 1 em $60 \%$ e a Filial SC 2 em 50\%. Conclui-se que a programação linear pode ser bastante útil para resolução de problemas empresariais que envolvam limitações ou restrições de 
Utilização da Programação Linear na Otimização de Resultados de uma Empresa do Ramo de Transporte Rodoviário de Cargas

Roberto Carlos Klann, Paulo Roberto da Cunha, Júlio Orestes da Silva, Jorge Eduardo Scarpin

recursos. No caso das empresas de transporte rodoviário de cargas, essa ferramenta pode ser interessante na distribuição de metas de volume transportado entre os estabelecimentos da empresa, auxiliando na distribuição dos veículos para cada estabelecimento, como apresentado nesse estudo.

Palavras-chave: Programação Linear. Otimização de Resultados. Transporte Rodoviário de Cargas.

\section{Use of Linear Programming in Optimal Results from a Company of Branch Load Road Transportation}

\section{ABSTRACT}

It deals the present article of a study in optimal results of a company of load road transport, that presents idle load capacity. This company is part of a highly competitive sector in Brazil, that needs managerial tools that can assist its managers in the process of decision taking. The objective of the article is to apply the linear programming to optimize the result of this company, established in the Region of the Valley of the Itajaí, Santa Catarina State. The study it is of exploratory character. The data had been gotten by means of documentary research, with consultation the primary and secondary documents of the company. The analysis is quantitative. The results point that the company needs increment the volume carried for its matrix and the two branch offices established in the State of Santa Catarina. However, had to the restrictions in the demand of each branch office, as well as the restriction of possessed vehicles for the company for this service, the matrix of the company should to increase its volume transported in approximately 17\%, Branch office SC 1 in 60\% and Branch office SC 2 in $50 \%$. One concludes that the linear programming can be sufficiently useful for resolution of problems that involve limitations or restrictions of resources. In the case of the companies of road load transport, this tool can be interesting in the distribution of goals of carried volume between the establishments of the company, assisting in the distribution of the vehicles for each establishment, as presented in this article.

Key Words: Linear programming. Optimal results. Load road transportation.

\section{INTRODUÇÃO}

O setor de transporte de cargas no Brasil, assim como outros ramos de atividade, tem enfrentado dificuldades financeiras, decorrentes principalmente da elevada concorrência do setor, agravada no final de 2008 e início de 2009 com a crise econômica mundial. 
Utilização da Programação Linear na Otimização de Resultados de uma Empresa do Ramo de Transporte Rodoviário de Cargas Roberto Carlos Klann, Paulo Roberto da Cunha, Júlio Orestes da Silva, Jorge Eduardo Scarpin

Bastos (2003) afirma que o transporte tem papel importante nas atividades das organizações, tanto na movimentação da matéria-prima como do produto final. O transporte rodoviário responde por $61,1 \%$ da movimentação de cargas no Brasil. São aproximadamente 1,94 milhões de caminhões e 340 mil cavalos mecânicos em atividade no país, espalhados por 1,6 milhões de rodovias federais, estaduais e municipais. A importância desse modal de transporte para o Brasil também se evidencia no número de empresas, 159 mil, contra apenas oito do modal ferroviário, 299 do aquaviário e 35 do modal aeroviário (CNT, 2009).

Neves (2009) comenta que as empresas em geral têm se esforçado para se manterem competitivas em seus mercados. Nesse contexto, a empresa de transporte de carga fracionada tem um papel importante como diferencial competitivo na transformação da cadeia logística. No entanto, para isso, as empresas de transporte precisam racionalizar e reduzir custos administrativos e operacionais. Além disso, o autor prevê um aumento na concorrência nesse setor no Brasil nos próximos dez anos.

Devido ao elevado nível de competitividade no transporte rodoviário de cargas, as empresas precisam criar e manter diferenciais competitivos, acrescentando novos processos de gestão, modernas tecnologias, ampliando sua área de abrangência no mercado. Por meio da potencialização e melhor utilização dos recursos envolvidos na atividade de transporte, as empresas de transporte rodoviário de cargas podem criar diferenciais frente à concorrência (BORGES NETO, 2004).

Nesse sentido, a programação linear para otimização de recursos escassos e maximização de resultados tem sido empregada com certa frequência. Como estudos sobre o tema podem ser citados o de Freitas, Henriques e Mansur (2003), que trata da comparação entre a programação linear e a Teoria das Restrições no cálculo do mix de produtos; Oenning et al. (2004), que abordam a Teoria das Restrições e a programação linear sob o enfoque da otimização da produção; Sott (2007) sobre o lucro incremental e a programação linear na tomada de decisões em ambiente de produção conjunta; entre outros.

Com aplicação mais específica ao setor de transporte rodoviário de cargas, a utilização da programação linear é tratada no trabalho de Berger et al. (2003), que 
Utilização da Programação Linear na Otimização de Resultados de uma Empresa do Ramo de Transporte Rodoviário de Cargas Roberto Carlos Klann, Paulo Roberto da Cunha, Júlio Orestes da Silva, Jorge Eduardo Scarpin

analisa a minimização de custos de transporte florestal com a utilização da programação linear; Seixas e Widmer (1993), que estudam a seleção e dimensionamento da frota de veículos rodoviários para o transporte principal de madeira utilizando-se de programação linear não inteira; entre outros.

Diante desse contexto, o objetivo desse artigo é aplicar a programação linear para otimizar o resultado de uma empresa de transporte rodoviário de cargas. Essa empresa está estabelecida na Região do Vale do Itajaí, Estado de Santa Catarina, possuindo duas filiais nesse estado e uma terceira filial no Estado de São Paulo.

O trabalho é justificado pela importância desse setor para o desenvolvimento econômico do país, em razão de ser o transporte rodoviário de cargas o principal modal utilizado pelas empresas brasileiras para movimentação de suas mercadorias. A aplicação da programação linear pode ajudar os gestores desse setor no processo de tomada de decisão, sobre quais custos reduzir, quais rotas implantar ou incrementar, entre outros.

O artigo está estruturado em seis tópicos. A introdução ao estudo apresenta uma breve contextualização e resultados de pesquisas recentes sobre o tema, além do objetivo da pesquisa. O segundo tópico traz uma abordagem sobre transporte rodoviário de cargas no Brasil, seu desenvolvimento histórico e alguns aspectos relacionados aos custos nesse setor. O terceiro tópico apresenta algumas considerações sobre a utilização da programação linear no processo de tomada de decisão. Na sequência apresenta-se o método e os procedimentos da pesquisa. $O$ tópico cinco apresenta a análise dos resultados da pesquisa, finalizando-se 0 artigo com as conclusões, seguidas das referências.

\section{TRANSPORTE RODOVIÁRIO DE CARGAS NO BRASIL}

O desenvolvimento do transporte inter-regional no Brasil se deu de maneira concomitante com o processo de industrialização no país, ao longo do século XX. "As relações de comércio das regiões periféricas, menos industrializadas, foram gradativamente se voltando principalmente para a região Sudeste, em detrimento do 
Utilização da Programação Linear na Otimização de Resultados de uma Empresa do Ramo de Transporte Rodoviário de Cargas Roberto Carlos Klann, Paulo Roberto da Cunha, Júlio Orestes da Silva, Jorge Eduardo Scarpin

comércio internacional. Floresceu também o comércio inter-regional entre os Estados do Sudeste e do Sul do país" (CASTRO, 2001, p. 35).

O crescimento do transporte de cargas, segundo Castro (2000), pode ser explicado por duas razões: (a) maior dispersão geográfica da atividade econômica no Brasil ao longo dos anos; e (b) crescimento marcante do comércio interno brasileiro, bem como da distância média percorrida por esses fluxos.

Borges Neto (2004) assinala que, no entanto, o desenvolvimento do setor de transporte rodoviário de cargas se concentrou em rotas que interligam os grandes centros urbanos, com ênfase no transporte entre capitais de maior relevância sob o ponto de vista econômico. Muitas empresas concentraram suas atividades a partir de São Paulo, devido a sua importância no cenário nacional.

Esse fato provocou uma proposta semelhante de serviços ofertados pelos diversos transportadores, resultando num nivelamento do mercado. Já alguns dos trechos que incluem municípios do interior dos Estados acabam não sendo atendidos de maneira regular pelas empresas, ocasionando aumento nos convênios e parcerias entre transportadores regionais menores para atender essa demanda.

Com isso, acabam se inserindo nesse mercado de carga fracionada diversas pequenas empresas de transporte e até transportadores autônomos, acirrando ainda mais a concorrência no setor. $O$ aumento do desemprego no país também contribui para essa situação, pois o transporte passa a ser uma opção interessante, devido à falta de exigência e de maior capacitação por parte do prestador do serviço.

Outro fator que fez aumentar a concorrência no setor foi a entrada no mercado de grandes players internacionais, com grandes investimentos e interessados em explorar o potencial do mercado brasileiro. Muitas empresas internacionais estão entrando para competir com as empresas locais, atuando como operadores logísticos (CARDOSO, 2001).

Essa concorrência acirrada faz com que as empresas de transporte rodoviário de cargas tenham que controlar de maneira ótima seus custos de operação. Segundo Bastos (2003, p. 41), "os valores que uma empresa deve pagar por serviços de transporte possuem ligação com os custos do serviço prestado". Isso significa que os 
Utilização da Programação Linear na Otimização de Resultados de uma Empresa do Ramo de Transporte Rodoviário de Cargas Roberto Carlos Klann, Paulo Roberto da Cunha, Júlio Orestes da Silva, Jorge Eduardo Scarpin

transportadores devem conhecer os seus custos para calcular o valor do frete, de maneira razoável a fim de se manterem competitivas no mercado.

Em relação ao transporte rodoviário de cargas, o custo é classificado em razão da distância percorrida, tendo como unidade variável a quilometragem percorrida. Diante disso, os custos ocorridos independentemente do deslocamento do caminhão são considerados fixos. Já aqueles que variam conforme as distâncias percorridas são classificadas como variáveis (OJIMA et al., 2007).

Já Bowersox e Closs (2001, p. 41) classificam como custos fixos aqueles "que não são influenciados diretamente pela quantidade de carga movimentada. São os custos de terminais, direitos de acesso, sistemas de informação e depreciação do veículo". Como custos variáveis os autores classificam aqueles que incluem "os custos diretos da transportadora ao transporte de cada carga. São geralmente referidas como custo/quilômetro ou por unidade de peso. Normalmente os custos dessa categoria são combustíveis, manutenção e algumas vezes mão-de-obra".

Como exemplos de custos fixos pode se citar a depreciação, despesas com pessoal (motorista), gastos com licenciamento do veículo, IPVA, seguro obrigatório, seguro do veículo e despesas administrativas. Como custos variáveis têm-se as despesas com combustíveis, lubrificantes e filtros, pneus, câmaras de ar, protetores e recapagens, pedágios e despesas de manutenção (peças e oficinas) (OJIMA et al., 2007).

Nota-se que a classificação em custos fixos e variáveis entre os autores citados possui algumas diferenças. Ojima et al. (2007) utilizam como parâmetro a distância percorrida, enquanto que Bowersox e Closs (2001) consideram o volume de carga transportada.

$\mathrm{Na}$ verdade, depende do que se pretende medir. Ao calcular tão somente o custo variável por quilômetro, a distância percorrida pelo veículo será o parâmetro a se considerar. No entanto, se o objetivo é obter o custo variável por quilômetro da tonelada transportada, será necessário considerar também o volume de carga transportado. Isso porque se um veículo transporta 10 ou 20 toneladas, o seu custo variável por 
Utilização da Programação Linear na Otimização de Resultados de uma Empresa do Ramo de Transporte Rodoviário de Cargas Roberto Carlos Klann, Paulo Roberto da Cunha, Júlio Orestes da Silva, Jorge Eduardo Scarpin

quilômetro será diluído pelo peso transportado. Dessa forma, o custo variável por quilômetro do quilo transportado será maior no transporte de dez toneladas.

De acordo com Bastos (2003), muitos custos de transportes são indivisíveis, dificultando a sua classificação. $O$ autor defende que as cargas transportadas variam em tamanho e peso no mesmo transporte, em se tratando de transporte de carga fracionada. Nesse caso, a alocação do custo para cada carga poderia ser atribuída pelo peso, pela metragem cúbica ou por outra base. Ballou (2001, p. 133) afirma que "não há fórmula simples para alocação de custos, e os custos de produção em uma base por carregamento permanece uma questão de julgamento".

Alguns fatores podem determinar variações significativas nos custos ou em sua composição, no caso do transporte rodoviário de cargas:

Quilometragem percorrida - quanto maior, menor o custo final por quilômetro, pois o custo fixo é dividido pela quilometragem;

Tipo de tráfego - em áreas urbanas o veículo consome mais combustível;

Tipo de via - as condições da rodovia influenciam os custos;

Região - dependendo do local onde a transportadora está instalada, pode haver variação no valor dos salários, combustíveis, impostos, entre outros;

Porte do veículo - quanto maior a capacidade de carga do veículo, menor o custo da tonelada por quilômetro;

Desequilíbrio nos fluxos - quando não há carga de retorno, ocorre um aumento no custo do transporte.

A partir do conhecimento de seus custos, a empresa transportadora pode definir estratégias de competição no mercado, como definição de tabelas de preços diferenciadas por clientes, tipos de rotas, entre outros. Além disso, poderá planejar o desenvolvimento de rotas alternativas, abertura de novas filiais ou incremento nas atividades de filiais já existentes. Nesse sentido, uma das ferramentas que podem auxiliar as empresas de transporte nessa tarefa é a programação linear. 
Utilização da Programação Linear na Otimização de Resultados de uma Empresa do Ramo de Transporte Rodoviário de Cargas Roberto Carlos Klann, Paulo Roberto da Cunha, Júlio Orestes da Silva, Jorge Eduardo Scarpin

\section{PROGRAMAÇÃO LINEAR}

A programação linear ( $P R L)$ é umas das técnicas utilizadas na Pesquisa Operacional, que consiste num método matemático utilizado no processo de tomada de decisão. A Pesquisa Operacional surgiu na Segunda Guerra Mundial, com o objetivo de resolver problemas relacionados às operações estratégicas e táticas militares (CORRAR; GARCIA, 2001).

Sobre a programação linear, Horngren (1981, p. 357) conceitua que

[...] é um método matemático poderoso para a solução de uma série de problemas empresariais com muitas variáveis em interação, que envolve, basicamente, a utilização de recursos limitados de forma a aumentar os lucros ou a diminuir o custo.

Quase sempre existem fatores limitadores ou recursos escassos que representam restrições ou limitações das alternativas existentes. Para Bregalda, Oliveira e Bornstein (1988, p. 61), trata-se de técnica matemática que tem por objetivo "encontrar a melhor solução para problemas que tenham seus modelos representados por expressões lineares".

Nossa e Chagas (1998) afirmam que o âmbito da PRL está restrito aos problemas cuja representação simbólica seja na forma de uma linha reta em um gráfico. Utilizam operações aritméticas simples, como: adição e subtração, além de funções pré-definidas, como: soma, tendência e previsão.

O modelo de programação linear é utilizado para auxiliar na resolução de problemas que envolvam alocações de recursos escassos, para atingir determinado objetivo. Esse problema pode ser resumido em: maximizar ou minimizar alguma variável dependente, que é função linear de diversas variáveis independentes, sujeitas a diversas restrições (CORRAR; GARCIA, 2001).

O modelo matemático de um problema de otimização pode ser formulado, segundo Garcia, Guerreiro e Corrar (1997), da seguinte maneira: 
Utilização da Programação Linear na Otimização de Resultados de uma Empresa do Ramo de Transporte Rodoviário de Cargas Roberto Carlos Klann, Paulo Roberto da Cunha, Júlio Orestes da Silva, Jorge Eduardo Scarpin

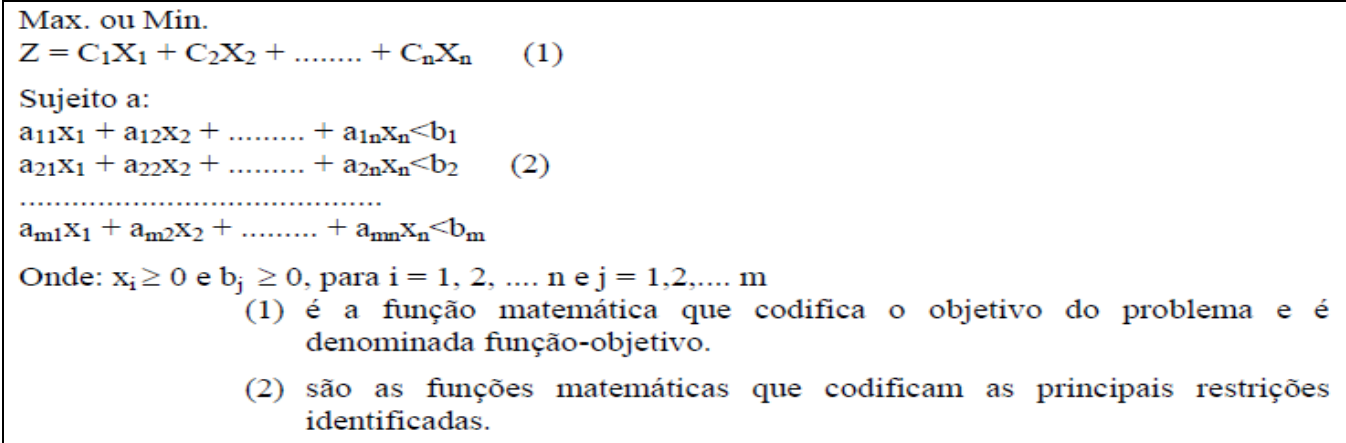

(1) é a função matemática que codifica o objetivo do problema e é denominada função-objetivo.

(2) são as funções matemáticas que codificam as principais restrições identificadas.

Figura 1 - Modelo matemático de programação linear

Fonte: Garcia, Guerreiro e Corrar (1997, apud OENNING et al., 2004, p. 213).

A linguagem simbólica utilizada na Figura 1 pode ser entendida da seguinte forma:

$\checkmark$ Z - função a ser minimizada ou maximizada;

$\checkmark$ xi - variáveis decisórias que representam as quantidades ou recursos que se quer otimizar;

$\checkmark$ ci-coeficientes de ganho ou custo que cada variável é capaz de gerar;

$\checkmark$ bj - quantidade disponível de cada recurso;

$\checkmark$ aij - quantidade de recurso que cada variável decisória consome (OENNING et al., 2004).

A construção de um modelo matemático, no caso do modelo linear, segundo Corrar e Garcia (2001, p. 6), pode ser facilitada seguindo-se os passos a seguir:

a) Determinar as variáveis de decisão;

b) Estabelecer o objetivo;

c) Determinar as relações básicas, especialmente restrições;

d) Calcular a solução ótima.

A planilha eletrônica Microsoft Excel®, com a utilização da ferramenta "Solver", permite a resolução de um problema de programação linear, bastando que o usuário defina as respostas para os três primeiros passos apresentados anteriormente (CORRAR; GARCIA, 2001). 
Utilização da Programação Linear na Otimização de Resultados de uma Empresa do Ramo de Transporte Rodoviário de Cargas Roberto Carlos Klann, Paulo Roberto da Cunha, Júlio Orestes da Silva, Jorge Eduardo Scarpin

Portanto, as empresas podem fazer uso da programação linear para resolver seus problemas de otimização de recursos, seja com o objetivo de redução de custos, otimização de resultados, ou qualquer outro processo de tomada de decisão gerencial.

As empresas de transporte rodoviário de cargas podem, então, aplicar a programação linear para redução de custos, escolha de rotas mais rentáveis, verificação de filiais ou praças onde a atividade da empresa deva ser incentivada, por produzir maior rentabilidade, entre outros. Para isso, podem fazer uso da planilha Microsoft Excel®, o que é apresentada na sequência.

\section{MÉTODO E PROCEDIMENTOS DE PESQUISA}

Nesse tópico são definidos o delineamento da pesquisa, com sua classificação quanto aos objetivos, procedimentos para coleta dos dados e quanto à abordagem.

\subsection{Delineamento da pesquisa}

A presente pesquisa caracteriza-se como exploratória, na medida em que este tipo de pesquisa é orientado para a descoberta, e não tem a intenção de testar hipóteses específicas. É útil na identificação de práticas inovadoras de produção e administração (HAIR JR et al., 2005).

Raupp e Beuren (2006) destacam que por meio da pesquisa exploratória buscase obter maior conhecimento sobre o assunto, contribuindo para esclarecer questões superficialmente abordadas sobre 0 assunto. Nesse sentido, esse artigo procura contribuir com maiores informações sobre a utilização da programação linear no processo de tomada de decisão nas empresas de transportes, auxiliando o processo gerencial da empresa.

Quanto ao procedimento técnico trata-se de uma pesquisa documental que, segundo Fachin (2006, p. 146), 
Utilização da Programação Linear na Otimização de Resultados de uma Empresa do Ramo de Transporte Rodoviário de Cargas Roberto Carlos Klann, Paulo Roberto da Cunha, Júlio Orestes da Silva, Jorge Eduardo Scarpin

corresponde a toda informação coletada, seja de forma oral, escrita ou visualizada. Ela consiste na coleta, classificação, seleção difusa e utilização de toda a espécie de informações, compreendendo também as técnicas e os métodos que facilitam a sua busca e a sua identificação.

Martins (2002, p. 35) a define como a pesquisa que "tem por finalidade reunir, classificar e distribuir os documentos de todo gênero dos diferentes domínios da atividade humana". Portanto, nesse estudo utilizou-se como fonte de dados os documentos gerenciais da empresa, coletados de forma oral, escrita e visualizada.

\subsection{Procedimentos de coleta e análise dos dados}

Nesse estudo utilizou-se documentos primários e secundários referentes ao ano de 2009, de uma empresa de transporte rodoviário de carga fracionada, que possui sua matriz localizada na Região do Vale do Itajaí, Estado de Santa Catarina. Essa empresa, que por questão de sigilo comercial, é denominada de "Transportes Alfa", possui duas filiais em Santa Catarina e outra no Estado de São Paulo.

A empresa em questão incorreu num dos problemas citados por Borges Neto (2004) no tópico dois. Concentrou suas atividades na cidade de São Paulo, utilizando a rota Santa Catarina - São Paulo e vice e versa. Atualmente, a empresa enfrenta o seguinte problema: possui três estabelecimentos em Santa Catarina (matriz e duas filiais) que precisam enviar diariamente um volume de carga para São Paulo. Trabalha atualmente com capacidade de carga ociosa, o que afeta sua rentabilidade.

A empresa precisa então determinar como essa capacidade ociosa pode ser distribuída entre os estabelecimentos de Santa Catarina, de modo a otimizar o seu resultado. Como restrições que a empresa está sujeita, pode-se citar a quantidade de veículos existentes para realizar o transporte (por decisão de política interna, a empresa não contrata veículos de terceiros), além da demanda estimada para cada estabelecimento.

Como diferenciais de custo de cada estabelecimento, o principal fator é a distância para a filial de São Paulo, que varia de estabelecimento para estabelecimento, ocasionando variação no custo variável do transporte. O preço do frete em cada 
Utilização da Programação Linear na Otimização de Resultados de uma Empresa do Ramo de Transporte Rodoviário de Cargas Roberto Carlos Klann, Paulo Roberto da Cunha, Júlio Orestes da Silva, Jorge Eduardo Scarpin

estabelecimento também é diferente. Portanto, a empresa precisa encontrar a relação ideal entre preço e distância, a fim de encontrar a melhor distribuição da capacidade ociosa.

Os dados sobre os custos fixos e variáveis, preços dos fretes, distância das filiais, quantidade de veículos disponíveis, entre outros, foram obtidos por meio dos documentos apresentados pela empresa em sua matriz, durante o mês de agosto de 2009, consultados in loco. Trata-se de documentos primários e secundários, como notas fiscais, planilhas e mapas de custos, manifestos de carga, entre outros.

Com base nos dados coletados, empregou-se o modelo de programação linear, por meio da ferramenta "Solver" do Microsoft Excel ${ }^{\circledR}$ para determinar a distribuição ótima da capacidade ociosa dos estabelecimentos da empresa localizados em Santa Catarina, a fim de maximizar o resultado global da empresa.

\section{DESCRIÇÃO E ANÁLISE DOS DADOS}

A empresa de Transportes Alfa possui um problema de otimização de recursos, como já explicado no tópico anterior. Para resolver esse problema, primeiramente foi necessário determinar seus custos fixos e variáveis.

Os custos variáveis da operação de transporte dos estabelecimentos de Santa Catarina para a Filial de São Paulo estão descritos na Tabela 1.

Os custos variáveis por quilômetro dos veículos da empresa compreendem os gastos com combustíveis, lubrificantes, lavagem e lubrificação e consumo de pneus. $O$ custo de combustível é obtido dividindo-se o preço por litro do óleo diesel pelo consumo médio dos veículos.

O custo com lubrificantes envolve o gasto com troca do óleo do cárter e trocar de óleo do câmbio. O cálculo é basicamente o mesmo do combustível. Multiplica-se o preço por litro do óleo pela quantidade de óleo a cada troca. O resultado é dividido pela quilometragem média percorrida entre uma troca e outra. 
Utilização da Programação Linear na Otimização de Resultados de uma Empresa do Ramo de Transporte Rodoviário de Cargas Roberto Carlos Klann, Paulo Roberto da Cunha, Júlio Orestes da Silva, Jorge Eduardo Scarpin

O custo de lavação e lubrificação do veículo por quilômetro é calculado dividindose o preço de uma lavação pela quilometragem percorrida entre uma lavação e outra, chegando-se num custo de $R \$ 0,1429$ por quilômetro rodado.

Por fim, o custo de reposição dos pneus envolve o valor do pneu novo, multiplicado pela quantidade de pneus do veículo (18), multiplicado por um índice de perda médio de $20 \%$, referentes a pneus estourados, cortados ou de qualquer forma danificados em viagem. Soma-se a isso o custo da recauchutagem, também multiplicado pela quantidade de pneus do veículo. Esses dois valores somados são então divididos pela quilometragem média percorrida por um pneu novo, recauchutado uma única vez, obtendo-se o custo de $\mathrm{R} \$ 0,2245$ por quilômetro. 
Utilização da Programação Linear na Otimização de Resultados de uma Empresa do Ramo de Transporte Rodoviário de Cargas Roberto Carlos Klann, Paulo Roberto da Cunha, Júlio Orestes da Silva, Jorge Eduardo Scarpin

Tabela 1 - Custos variáveis por km da operação de transporte

\begin{tabular}{|c|c|c|}
\hline \multicolumn{3}{|c|}{ Custos Variáveis por $\mathrm{Km}$} \\
\hline 1. Combustível & \multicolumn{2}{|c|}{$\mathbf{R} \$$} \\
\hline a) Preço do combustível por litro & $\mathbf{R} \$$ & 2,45 \\
\hline b) Rendimento do combustivel ( $\mathrm{Km} / \mathrm{litro})$ & & 2,80 \\
\hline c) Combustível (a:b) & $\mathbf{R} \$$ & 0,8750 \\
\hline 2. Lubrificantes & \multicolumn{2}{|c|}{$\mathbf{R} \$$} \\
\hline a) Preço do óleo do cárter (por litro) & $\mathbf{R} \mathbf{S}$ & 10,00 \\
\hline b) Capacidade de óleo do cárter (litros) & & 35 \\
\hline c) Reposição até a próxima troca (litros) & & 2 \\
\hline d) Troca de óleo do cárter $(\mathrm{Km})$ & & 20.000 \\
\hline e) Preço do óleo de câmbio/diferencial (por litro) & $\mathbf{R} \mathbf{3}$ & 15,00 \\
\hline f) Capacidade de óleo do diferencial (litros) & & 15 \\
\hline g) Troca de óleo do diferencial (Km) & & 80.000 \\
\hline h) Lubrificantes $((\mathrm{a} \times(\mathrm{b} \times \mathrm{c})): \mathrm{d})+((\mathrm{e} \times \mathrm{f}): \mathrm{g})$ & $\mathbf{R} \$$ & $\mathbf{0 , 0 3 7 8}$ \\
\hline 3. Lavagem e Lubrificação & \multicolumn{2}{|c|}{$\mathbf{R} \mathbf{S}$} \\
\hline a) Preço da Lavagem do veículo & RS & 100,00 \\
\hline b) Periodicidade da lavagem $(\mathrm{Km})$ & & 700 \\
\hline c) Lavagem e Lubrificação (a : b) & $\mathbf{R} \mathbf{S}$ & 0,1429 \\
\hline 4. Pneus & \multicolumn{2}{|c|}{$\mathbf{R} \mathbf{S}$} \\
\hline a) Valor do pneu & $\mathbf{R} \$$ & $1.080,00$ \\
\hline b) Valor da câmara & $\mathbf{R} \mathbf{S}$ & - \\
\hline c) Valor da recauchtagem & $\mathbf{R} \mathbf{S}$ & 450,00 \\
\hline d) Quantidade de pneus & & 18 \\
\hline e) Perda do pneu novo & & 1,20 \\
\hline f) Vida útil do pneu (Km) & & 140.000 \\
\hline g) Pneus $(((((a+b) \times d) \times e)+((c \times d)): f)$ & $\mathbf{R} \mathbf{S}$ & 0,2245 \\
\hline Custos variáveis por $\mathrm{Km}(1+2+3+4)$ & $\mathbf{R} \$$ & $\mathbf{1 , 2 8 0 2}$ \\
\hline
\end{tabular}

O custo variável total por quilômetro é o resultado da soma de todos esses custos descritos, que para a empresa de Transportes Alfa alcança o valor de $R \$$ 1,2802 .

Após o cálculo do custo variável por quilômetro, é necessário também obter o valor do custo fixo mensal da empresa, apresentado na Tabela 2.

Os custos fixos mensais envolvem os valores de depreciação do veículo, salário do motorista, gastos com o emplacamento do veículo, seguro e demais custos e despesas fixas. 
Utilização da Programação Linear na Otimização de Resultados de uma Empresa do Ramo de Transporte Rodoviário de Cargas

Roberto Carlos Klann, Paulo Roberto da Cunha, Júlio Orestes da Silva, Jorge Eduardo Scarpin

A depreciação é obtida a partir do valor do cavalo mecânico mais a carreta. Desse valor desconta-se o valor residual do veículo (valor estimado de venda ao final de sua vida útil). Desconta-se também o valor dos pneus, que são considerados custos variáveis, apresentados na Tabela 1. Dessa forma, obtém-se o valor depreciável do conjunto (cavalo mecânico mais carreta), que é dividido pelo prazo de vida útil estimado pela empresa (5 anos), que por sua vez é dividido por 12 meses, chegando-se então no custo mensal de depreciação do veículo. 
Utilização da Programação Linear na Otimização de Resultados de uma Empresa do Ramo de Transporte Rodoviário de Cargas Roberto Carlos Klann, Paulo Roberto da Cunha, Júlio Orestes da Silva, Jorge Eduardo Scarpin

Tabela 2 - Custos fixos mensais

\begin{tabular}{|c|c|c|}
\hline \multicolumn{3}{|l|}{ Custos fixos mensais } \\
\hline 1. Depreciação & & RS \\
\hline a) valor do veículo mais carreta (conjunto) & $\mathbf{R} \$$ & $380.000,00$ \\
\hline b) valor residual do conjunto & $-\mathbf{R} \$$ & $95.000,00$ \\
\hline c) Valor depreciável do conjunto (a - b) & $\mathbf{R} \mathbf{S}$ & $285.000,00$ \\
\hline d) Quantidade de pneus & & 18 \\
\hline e) Valor do pneu & $\mathbf{R} \$$ & $1.300,00$ \\
\hline f) Valor da câmara de ar & $\mathbf{R} \$$ & - \\
\hline g) Valor do estepe & $\mathbf{R} \$$ & $1.300,00$ \\
\hline h) Valor total dos pneus $((d \times(e+f))+g)$ & $\mathbf{R} \$$ & $24.700,00$ \\
\hline i) Valor depreciável do conjunto sem os pneus $(c-h)$ & $\mathbf{R} \$$ & $262.900,00$ \\
\hline j) Tempo de vida útil do veículo (anos) & & 5 \\
\hline k) Custo de depreciação mensal ( $\mathrm{i}: \mathrm{j}: 12$ meses) & $\mathbf{R} \$$ & $4.381,67$ \\
\hline 2. Salário do motorista & & $\mathbf{R S}$ \\
\hline a) Salário do motorista no ano (11 meses) & $\mathbf{R} \$$ & $14.000,00$ \\
\hline b) Férias mais $1 / 3$ & $\mathbf{R} \mathbf{S}$ & $1.696,93$ \\
\hline c) 13o. Salário & $\mathbf{R} \$$ & $1.272,73$ \\
\hline d) Remuneração anual $(\mathbf{a}+\mathbf{b}+\mathbf{c})$ & $\mathbf{R} \$$ & $16.969,65$ \\
\hline e) Encargos sociais (d x 36,8\%) & $\mathbf{R} \$$ & $6.244,83$ \\
\hline f) Custo total anual da mão-de-obra $(d+e)$ & $\mathbf{R} \$$ & $23.214,49$ \\
\hline g) Custo da mão-de-obra mensal (f : 12 meses) & $\mathbf{R} \mathbf{S}$ & $1.934,54$ \\
\hline 3. IPVA, Seguro obrigatório e Licenciamento anual & & RS \\
\hline a) Valor anual médio por veículo & $\mathbf{R} \$$ & $4.200,00$ \\
\hline b) Custo médio mensal (a : 12 meses) & $\mathbf{R} \$$ & 350,00 \\
\hline 4. Seguro do veículo & & $\mathbf{R S}$ \\
\hline a) valor médio do seguro anual & $\mathbf{R} \mathbf{\$}$ & $7.200,00$ \\
\hline b) Custo médio mensal (a : 12 meses) & $\mathbf{R} \$$ & 600,00 \\
\hline 5. Demais custos e despesas fixas & & $\mathbf{R S}$ \\
\hline a) Valor médio dos demais custos e despesas fixas mensais & $\mathbf{R} \$$ & $7.200,00$ \\
\hline b) Custo médio mensal por veículo (a : 8 veículos) & $\mathbf{R} \$$ & 900,00 \\
\hline 6. Custo fixo mensal por veículo $(1+2+3+4+5)$ & $\mathbf{R} \$$ & $8.166,21$ \\
\hline
\end{tabular}

O custo com salário do motorista leva em conta o gasto anual com salários, férias, 13ํ. salário e encargos. Esses valores são então divididos por 12 meses, obtendo-se o custo mensal com salário.

O custo de licenciamento do veículo leva em conta os gastos com IPVA, seguro obrigatório, taxas e pagamento de despachante. A soma desses valores é dividida por 
Utilização da Programação Linear na Otimização de Resultados de uma Empresa do Ramo de Transporte Rodoviário de Cargas Roberto Carlos Klann, Paulo Roberto da Cunha, Júlio Orestes da Silva, Jorge Eduardo Scarpin

12 meses, chegando-se no custo mensal com licenciamento. Para o seguro do veículo e demais gastos e despesas fixas o cálculo é o mesmo. O custo fixo mensal por veículo envolve a soma de todos os valores descritos na Tabela 2, totalizando $\mathrm{R} \$$ 8.166,21.

O cenário atual da empresa, quanto ao volume transportado por Filial de Santa Catarina para a Filial de São Paulo, bem como os dados de custos fixos e variáveis, é apresentado na Tabela 3.

Tabela 3 - Cenário atual da empresa

\begin{tabular}{|c|c|c|c|c|c|c|c|c|}
\hline Itens & \multicolumn{2}{|c|}{ Matriz } & \multicolumn{2}{|c|}{ Filial SC 1} & \multicolumn{2}{|c|}{ Filial SC 2} & \multicolumn{2}{|r|}{ Total } \\
\hline a) peso transportado $(\mathrm{Kg})$ & \multicolumn{2}{|r|}{770.000} & \multicolumn{2}{|r|}{250.000} & \multicolumn{2}{|r|}{200.000} & \multicolumn{2}{|r|}{1.220 .000} \\
\hline b) preço por $\mathrm{kg}$ & RS & $\mathbf{0 , 2 5}$ & RS & $\mathbf{0 , 2 3}$ & RS & $\mathbf{0 , 2 7}$ & & \\
\hline c) capacidade de carga por veículo $(\mathrm{Kg})$ & & 25.000 & & 25.000 & & 25.000 & & \\
\hline d) Custos variáveis por $\mathrm{Km}$ & RS & 1,2802 & RS & 1,2802 & RS & 1,2802 & & \\
\hline e) Distância da viagem (Km) & \multicolumn{2}{|r|}{700} & \multicolumn{2}{|c|}{667} & \multicolumn{2}{|c|}{770} & & \\
\hline f) Custos variáveis por viagem & RS & 896,11 & RS & 853,86 & RS & $\mathbf{9 8 5 , 7 2}$ & & \\
\hline g) Custos variáveis por $\mathrm{Kg}$ & RS & $\mathbf{0 , 0 3 5 8}$ & RS & $\mathbf{0 , 0 3 4 2}$ & RS & 0,0394 & & \\
\hline f) Número de viagens & \multicolumn{2}{|r|}{31} & \multicolumn{2}{|r|}{10} & \multicolumn{2}{|c|}{8} & & 49 \\
\hline i) margem de contribuição por $\mathrm{Kg}$ & RS & 0,2142 & RS & $\mathbf{0 , 1 9 5 8}$ & RS & 0,2306 & & \\
\hline j) Margem de contribuição total & RS & $164.899,85$ & RS & $48.961,36$ & RS & 46.114,24 & RS & $259.975,46$ \\
\hline \multicolumn{7}{|c|}{ k) custos fixos mensais. } & RS & $65.329,66$ \\
\hline \multicolumn{7}{|c|}{ 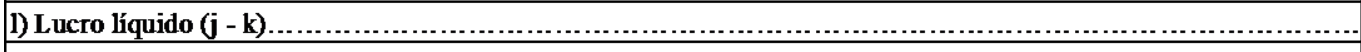 } & RS & $194.645,80$ \\
\hline capacidade de carga mensal $(\mathrm{kg})$ & & 900.000 & & 500.000 & & 300.000 & & 1.700 .000 \\
\hline peso mínimo mensal a transportar (kg) & & 100.000 & & 100.000 & & 100.000 & & 300.000 \\
\hline custo fixo mensal por veículo & RS & $8.166,21$ & & & & & & \\
\hline número máximo de viagens & & 64 & & & & & & \\
\hline
\end{tabular}

A Tabela 3 revela que a empresa transporta atualmente 1.220 toneladas mensais para a Filial São Paulo. No entanto, pela demanda estimada pela empresa, seria possível transportar até 1.700 toneladas. Além disso, a empresa tem capacidade para realizar 64 viagens mensais de SC para SP. Atualmente está realizando apenas 49 viagens em média. Verifica-se que a empresa possui uma capacidade de transporte ociosa de aproximadamente $30 \%$.

O preço do frete por quilo varia para cada estabelecimento. Como a empresa utiliza veículos de porte padrão, a capacidade de carga por viagem é a mesma para todos os estabelecimentos ou viagens. O custo variável por quilo é o obtido na Tabela 1. A distância da viagem compreende a quilometragem percorrida a partir de cada 
Utilização da Programação Linear na Otimização de Resultados de uma Empresa do Ramo de Transporte Rodoviário de Cargas Roberto Carlos Klann, Paulo Roberto da Cunha, Júlio Orestes da Silva, Jorge Eduardo Scarpin

estabelecimento de SC até a Filial São Paulo, variando, portanto, para cada estabelecimento.

Os custos variáveis por viagem são obtidos multiplicando-se o custo variável por quilômetro pela distância percorrida em cada viagem. Dividindo esse valor pela capacidade de carga de cada veículo, chega-se ao custo variável por quilo transportado (item g da Tabela 3).

Ao subtrair do preço do frete por quilo esse custo variável, chega-se na margem de contribuição por quilo (i), que multiplicado pelo peso transportado (a), resulta na margem de contribuição total de cada estabelecimento (j).

O custo fixo total $(k)$ é obtido pela multiplicação do custo fixo mensal por veículo (Tabela 2) pela quantidade de veículos possuídos pela empresa (oito veículos). A margem de contribuição total é diminuída desse custo fixo mensal total, resultando no lucro líquido da matriz e filiais de Santa Catarina.

Como a empresa apresenta capacidade de transporte ociosa, esse lucro pode ser maximizado. O problema é considerar as restrições de demanda de cada estabelecimento, mais a restrição do número de viagens máximo, pois a empresa não contrata serviços de transporte terceirizados.

Para encontrar a solução ótima, aplica-se na sequência a ferramenta Solver do Microsoft Excel®. Essa ferramenta permite encontrar um valor ideal para uma fórmula em uma célula, chamada de célula de destino, em uma planilha eletrônica. O Solver ajusta os valores nas células variáveis especificadas para produzir o resultado desejado. A entrada dos dados no software é realizada conforme a Figura 2 a seguir.

$\mathrm{Na}$ sequência escolhe-se a opção "Dados/Solver" do Microsoft Excel ${ }^{\circledR} 2007$, seleciona-se a célula de destino, as células variáveis e as restrições, conforme a Figura 3. 
Utilização da Programação Linear na Otimização de Resultados de uma Empresa do Ramo de Transporte Rodoviário de Cargas Roberto Carlos Klann, Paulo Roberto da Cunha, Júlio Orestes da Silva, Jorge Eduardo Scarpin

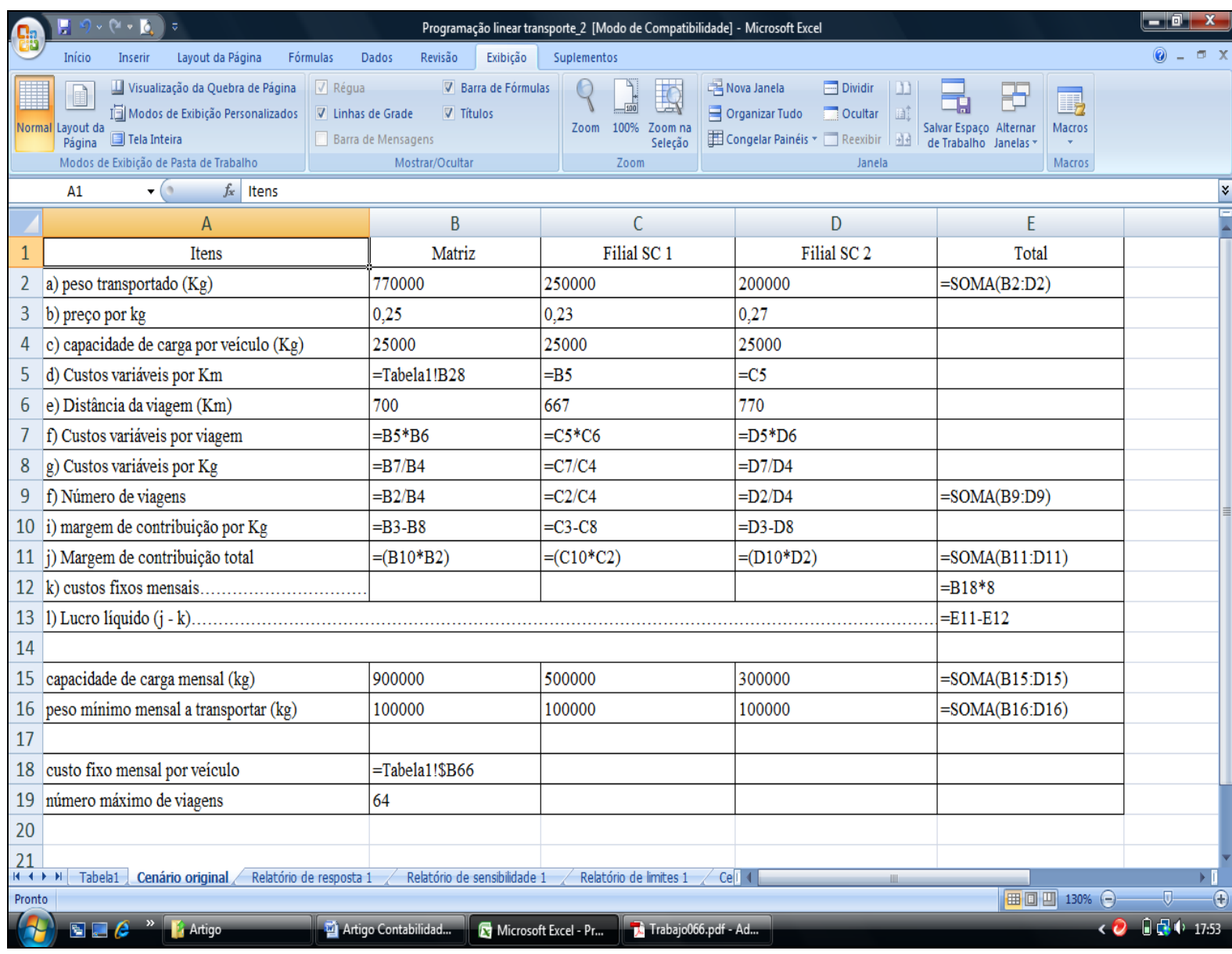

Figura 2 - Entrada de dados do cenário atual da empresa no Microsoft Excel ${ }^{\circledR} 2007$

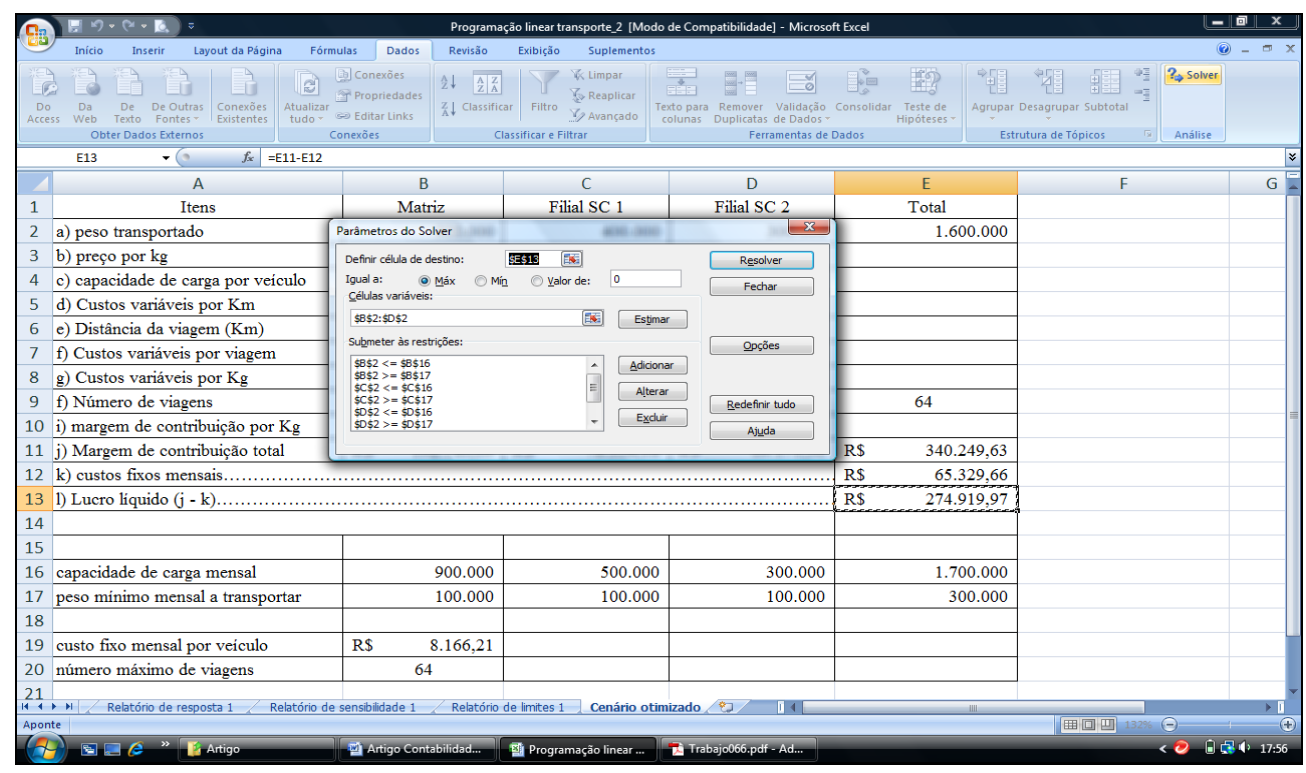

Figura 3 - Entrada de dados no Solver do Microsoft Excel ${ }^{\circledR} 2007$ 
Utilização da Programação Linear na Otimização de Resultados de uma Empresa do Ramo de Transporte Rodoviário de Cargas Roberto Carlos Klann, Paulo Roberto da Cunha, Júlio Orestes da Silva, Jorge Eduardo Scarpin

Como célula de destino foi selecionada a célula E13 (Lucro líquido). As células variáveis foram definidas como as células B2, C2 e D2 (peso transportado). Como restrições foram relacionadas as seguintes:
a) b2 $\leq$ b16 - peso transportado da matriz menor ou igual a 900.000 quilos;
b) b2 $\geq$ b17 - peso transportado da matriz maior ou igual a 100.000 quilos;
c) c2 $\leq$ c16 - peso transportado da Filial SC 1 menor ou igual a 500.000 quilos;
d) c2 $\geq$ c17 - peso transportado da Filial SC 1 maior ou igual a 100.000 quilos;
e) d2 $\leq$ d16 - peso transportado da Filial SC 2 menor ou igual a 300.000 quilos;
f) d2 $\geq$ d17 - peso transportado da Filial SC 2 maior ou igual a 100.000 quilos;
g) e2 $\leq$ e 16 - peso total transportado menor ou igual a 1.700 .000 quilos;
h) e9 $\leq$ b20 - número total de viagens mensais inferior ou igual a 64 .

Após a seleção dessas células, pode-se entrar no botão "opções" na caixa do Solver para selecionar algumas opções de cálculo, conforme a Figura 4.

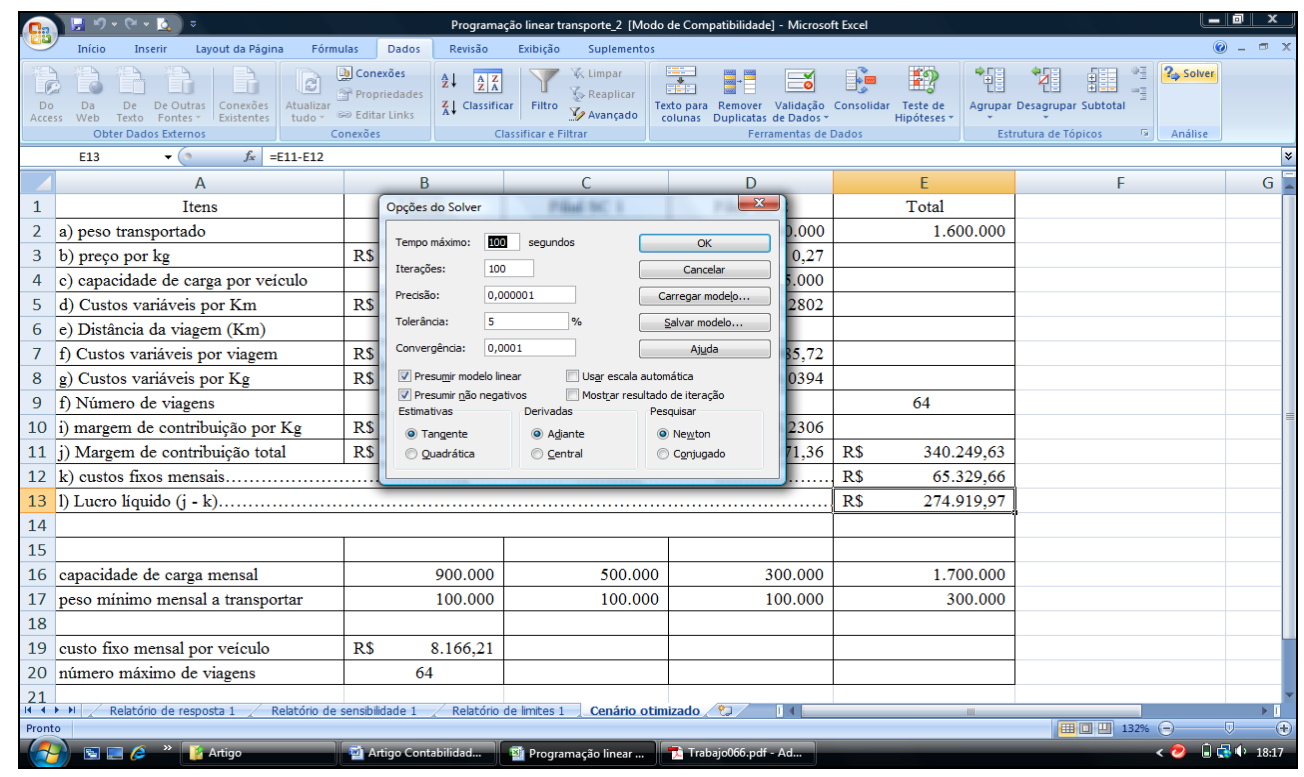

Figura 4 - Caixa de opções do Solver do Microsoft Excel ${ }^{\circledR} 2007$

Duas informações importantes devem ser selecionadas nessa caixa. A primeira é presumir modelo linear. A segunda, como se está trabalhando com a escolha de resultado ótimo, é importante "presumir não negativos", pois caso contrário o software 
Utilização da Programação Linear na Otimização de Resultados de uma Empresa do Ramo de Transporte Rodoviário de Cargas Roberto Carlos Klann, Paulo Roberto da Cunha, Júlio Orestes da Silva, Jorge Eduardo Scarpin

pode selecionar a opção com prejuízo como resultado desejado. As demais opções são default do próprio software.

Após selecionar essas opções, deve-se escolher a opção "resolver" e então escolher os relatórios de saída, conforme a Figura 5.

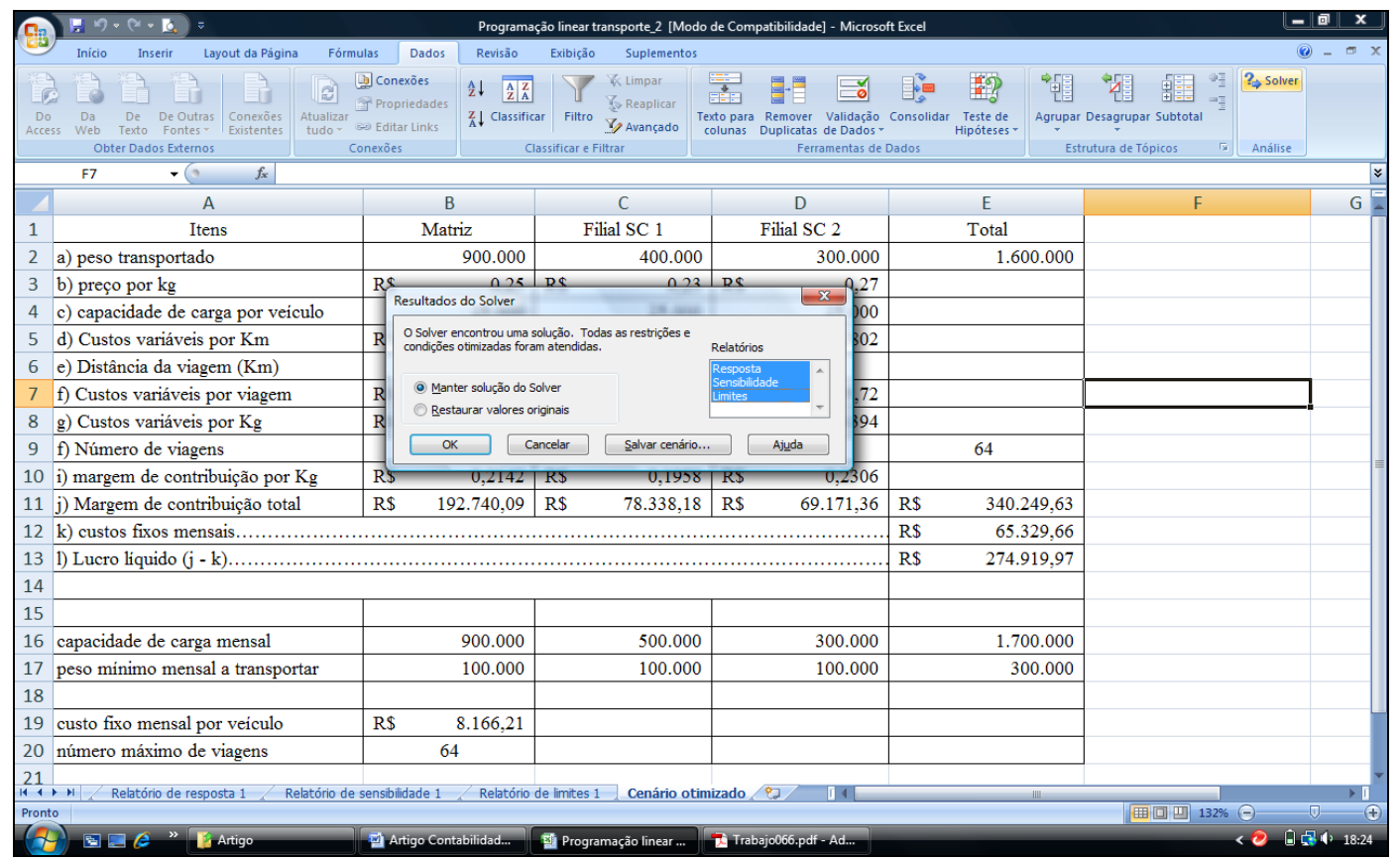

Figura 5 - Caixa de resultados do Solver do Microsoft Excel ${ }^{\circledR} 2007$

Pode-se selecionar três relatórios de saída do Solver, o de Resposta, o de Sensibilidade e o de Limites. O Relatório de Resposta é apresentado na Figura 6. 
Utilização da Programação Linear na Otimização de Resultados de uma Empresa do Ramo de Transporte Rodoviário de Cargas Roberto Carlos Klann, Paulo Roberto da Cunha, Júlio Orestes da Silva, Jorge Eduardo Scarpin

\begin{tabular}{|c|c|c|c|c|c|}
\hline \multicolumn{6}{|c|}{$\begin{array}{l}\text { Microsoft Excel 12.0 Relatório de resposta } \\
\text { Planilha: [Programaço Einear transporte_2xk]Cenário otimizado } \\
\text { Relatório criado: 05/09/2009 18:28:58 }\end{array}$} \\
\hline \multicolumn{6}{|c|}{ Célula de destino (Máx) } \\
\hline Céluda & Nome & Valor original & Valor final & & \\
\hline \$E\$13 & l) Lucro líquido (j- k) & R\$ $194.645,80$ & R\$ $274.919,97$ & & \\
\hline \multicolumn{6}{|c|}{ Células ajustáveis } \\
\hline Cólula & Nome & Valor original & Valor final & & \\
\hline \$B\$2 & a) peso transportado Matrìz & 770.000 & 900.000 & & \\
\hline$\$ C \$ 2$ & a) peso transportado Filial SC 1 & 250.000 & 400.000 & & \\
\hline \$D\$2 & a) peso transportado Filial SC 2 & 200.000 & 300.000 & & \\
\hline \multicolumn{6}{|l|}{ Restriçöes } \\
\hline Cólula & Nome & Valor da céflula & Fómula & Status & Transigência \\
\hline \$E\$9 & f) Número de viagens Total & 64 & $\$ E \$ 9<=\$ B \$ 20$ & Agrupar & 0 \\
\hline \$E\$2 & a) peso transportado Total & 1.600 .000 & $\$ E \$ 2<=\$ E \$ 16$ & Sem agrupar & 100000 \\
\hline \$D\$2 & a) peso transportado Filial SC 2 & 300.000 & $\$ D \$ 2>=\$ D \$ 17$ & Sem agrupar & 200.000 \\
\hline \$BS2 & a) peso transportado Matriz & 900.000 & $\$ B \$ 2>\subset \$ B \$ 17$ & Sem agrupar & 800.000 \\
\hline$\$ C \$ 2$ & a) peso transportado Filial SC 1 & 400.000 & $\$ C \$ 2>=\$ C \$ 17$ & Sem agrupar & 300.000 \\
\hline \$B\$2 & a) peso transportado Matriz & 900.000 & $\$ B \$ 2<=\$ B \$ 16$ & Agrupar & 0 \\
\hline$\$ C \$ 2$ & a) peso transportado Filial SC 1 & 400.000 & $\$ C \$ 2<=\$ C \$ 16$ & Sem agrupar & 100000 \\
\hline \$D\$2 & a) peso transportado Filial SC 2 & 300.000 & $\$ D \$ 2<=\$ D \$ 16$ & Agrupar & 0 \\
\hline
\end{tabular}

Figura 6 - Relatório de resposta 1 do Solver do Microsoft Excel ${ }^{\circledR} 2007$

O Relatório de Resposta indica o valor do lucro líquido maximizado de $R \$$ 274.919,97 (célula de destino), além da variação no peso transportado por estabelecimento (células ajustáveis).

Nota-se nesse relatório que a matriz deverá aumentar o seu peso transportado para 900 toneladas/mês (+17\%), a Filial SC 1 para 400 toneladas/mês $(+60 \%)$ e a Filial SC 2 para 300 toneladas/mês (+ 50\%). Quanto às restrições, o relatório apresenta ainda uma ociosidade no peso total transportado, na coluna transigência, de 100 toneladas, que está limitado à capacidade de viagens da empresa (64), que foi plenamente atingida.

Na Figura 7 apresenta-se o Relatório de Sensibilidade, que indica o valor final a ser transportado por cada estabelecimento, além de algumas informações sobre as variações no resultado provocadas pelas restrições. 
Utilização da Programação Linear na Otimização de Resultados de uma Empresa do Ramo de Transporte Rodoviário de Cargas Roberto Carlos Klann, Paulo Roberto da Cunha, Júlio Orestes da Silva, Jorge Eduardo Scarpin

\begin{tabular}{|c|c|c|c|c|c|}
\hline \multicolumn{6}{|c|}{$\begin{array}{l}\text { Microsoft Excel 12.0 Relatório de sensibilidade } \\
\text { Planilha: [Programação linear transporte_2.xls]Cenário otimizado } \\
\text { Relatório criado: 05/09/2009 18:28:58 }\end{array}$} \\
\hline \multicolumn{6}{|l|}{ Células ajustáveis } \\
\hline Célula & $\begin{array}{l}\text { Final } \\
\text { Valor }\end{array}$ & $\begin{array}{c}\text { Reduzido } \\
\text { Custo }\end{array}$ & $\begin{array}{c}\text { Objetivo } \\
\text { Coeficiente }\end{array}$ & $\begin{array}{l}\text { Permissível } \\
\text { Acréscimo }\end{array}$ & $\begin{array}{l}\text { Permissível } \\
\text { Decréscimo }\end{array}$ \\
\hline \$B\$2 a) peso transportado Matriz & 900.000 & $\mathbf{0}$ & 0,21415565 & $1 \mathrm{E}+30$ & 0,018310195 \\
\hline$\$ C \$ 2$ a) peso transportado Filial SC 1 & 400.000 & $\mathbf{0}$ & 0,195845455 & 0,018310195 & 0,195845455 \\
\hline \$D\$2 a) peso transportado Filial SC 2 & 300.000 & $\mathbf{0}$ & 0,230571215 & $1 E+30$ & 0,03472576 \\
\hline \multicolumn{6}{|l|}{ Restrições } \\
\hline Célula & $\begin{array}{l}\text { Final } \\
\text { Valor }\end{array}$ & $\begin{array}{l}\text { Sombra } \\
\text { Preço }\end{array}$ & $\begin{array}{c}\text { Restrição } \\
\text { Lateral R.H. }\end{array}$ & $\begin{array}{l}\text { Permissível } \\
\text { Acréscimo }\end{array}$ & $\begin{array}{l}\text { Permissivel } \\
\text { Decréscimo }\end{array}$ \\
\hline \$E\$9 f) Número de viagens Total & 64 & 4.896 & 64 & 4 & 12 \\
\hline \$E\$2 a) peso transportado Total & 1.600 .000 & - & 1700000 & $1 E+30$ & 100000 \\
\hline
\end{tabular}

Figura 7 - Relatório de sensibilidade do Solver do Microsoft Excel ${ }^{\circledR} 2007$

O Relatório de Sensibilidade, na parte das células ajustáveis, apresenta o valor final do peso a ser transportado por estabelecimento para a otimização do resultado da empresa. Os valores de "Permissível Acréscimo" e "Permissível Decréscimo" correspondem aos intervalos de variação para a margem de contribuição de cada estabelecimento.

Em relação às restrições desse relatório, a coluna "Sombra Preço" corresponde ao valor que a empresa deixa de ganhar por viagem a mais, por não dispor de mais um veículo para realizar esse transporte. As colunas "Permissível Acréscimo" e "Permissível Decréscimo" fornecem intervalos de variação para a quantidade de recursos sem que se mude a relação de crescimento (sombra preço) no ganho total obtido pela solução ótima.

\section{CONCLUSÃO}

O objetivo deste artigo foi aplicar a programação linear para otimizar o resultado de uma empresa de transporte rodoviário de cargas, estabelecida na Região do Vale do Itajaí, cuja análise de dados permite concluir: 
Utilização da Programação Linear na Otimização de Resultados de uma Empresa do Ramo de Transporte Rodoviário de Cargas Roberto Carlos Klann, Paulo Roberto da Cunha, Júlio Orestes da Silva, Jorge Eduardo Scarpin

A empresa possui capacidade de carga ociosa, quanto ao transporte de sua matriz e duas filiais, instaladas no Estado de Santa Catarina, com destino a sua filial no Estado de São Paulo. Esse fato provoca uma limitação na sua lucratividade, que poderia ser incrementada caso eliminasse essa ociosidade.

No entanto, para que consiga resolver esse problema, os gestores da empresa precisam considerar duas limitações ou restrições, a de demanda de cada estabelecimento e a quantidade limitada de veículos para realizar esse transporte.

Com a utilização da programação linear, por meio da ferramenta Solver do Microsoft Excel®, pode-se chegar ao valor ideal que poderia ser transportado por filial, de modo a otimizar o resultado. Considerando as restrições já comentadas, o software ofereceu um cenário ótimo para a empresa, que maximiza o seu resultado em $R \$$ 274.919,97 (Figura 6).

Conclui-se, dessa forma, que a programação linear pode ser bastante útil para resolução de problemas empresariais que envolvam limitações ou restrições de recursos. No caso das empresas de transporte rodoviário de cargas, essa ferramenta pode ser interessante na distribuição de metas de volume transportado entre os seus estabelecimentos, auxiliando na distribuição dos veículos para cada estabelecimento, como apresentado nesse artigo.

No entanto, é necessário o acompanhamento constante do desenvolvimento do modelo, para que possa ser ajustado sempre que ocorram alterações, seja de demanda, de recursos, ou em ambos.

\section{REFERÊNCIAS}

BASTOS, Isabela D. (2003). Avaliação do desempenho logístico do serviço de transporte rodoviário de cargas - um estudo de caso no setor de revestimentos cerâmicos. 2003. 168 f. (Dissertação de Mestrado). Universidade Federal de Santa Catarina - UFSC, Florianópolis.

BERGER, Ricardo et al. (2003). Minimização do custo de transporte florestal com a utilização da programação linear. Revista Floresta, 33 (1), p. 53-62, jun.

BORGES NETO, Apparício. (2004). A terceirização como alternativa para a ampliação da área de distribuição de uma empresa de transporte rodoviário de cargas. 83 f. 
Utilização da Programação Linear na Otimização de Resultados de uma Empresa do Ramo de Transporte Rodoviário de Cargas Roberto Carlos Klann, Paulo Roberto da Cunha, Júlio Orestes da Silva, Jorge Eduardo Scarpin

(Dissertação de Mestrado). Escola de Engenharia, Universidade Federal do Rio Grande do Sul - UFRGS, Porto Alegre.

BREGALDA, Paulo Fábio; OLIVEIRA, Antonio A. F. de; BORNSTEIN, Cláudio T. (1988). Introdução à programação linear. (3 ed.). Rio de Janeiro: Campus.

CARDOSO, Fátima. (2001). Operadores Logísticos: tendência é de crescimento ordenado. Revista Tecnologística, São Paulo, n. 67, jun.

CONFEDERAÇÃO NACIONAL DO TRANSPORTE - CNT. (2009). Boletim estatístico. Disponível

http://www.cnt.org.br/portal/img/arquivos/Boletim\%20Estatístico\%20CNT\%20-

\%202009.pdf. Acesso em 02/set/2009.

CORRAR, Luis João; GARCIA, Editinete A. da Rocha. (2001). Programação linear: uma aplicação à contabilidade de custos no processo de tomada de decisão. In: CONGRESSO INTERNACIONAL DE CUSTOS, 7, León. Disponível em: http://www.intercostos.org/documentos/Trabajo066.pdf. Acesso em 04/set/2009.

FACHIN, Odília. (2006). Fundamentos de metodologia. (5 ed.). São Paulo: Saraiva.

FREITAS, Angelo A. de Miranda; HENRIQUES, Flávio C.; MANSUR, Rodrigo T. (2003). Comparação entre programação linear e teoria das restrições no cálculo do mix de produtos: um exemplo real. In: ENCONTRO NACIONAL DE ENGENHARIA DE PRODUÇÃO - ENEGEP. 23, Outro Preto. Disponível em http://www.abepro.org.br/biblioteca/ENEGEP2003_TR0101_0346.pdf. Acesso em: 03/set/2009.

HAIR JR., Joseph F. et al. (2005). Fundamentos de métodos de pesquisa em administração. Porto Alegre: Bookman.

HORNGREN, Charles T. (1981). Introdução à contabilidade gerencial. (5 ed.). Rio de Janeiro: LTC.

MARTINS, Gilberto de Andrade. (2002). Manual para elaboração de monografias e dissertações. (3 ed.). São Paulo: Atlas.

NEVES, Marco Antonio Oliveira. Tendências no transporte rodoviário de cargas fracionadas no Brasil. Disponível em: http://www.cnt.org.br/portal/webcnt/artigoEntrevista.aspx?id=3ab715b2-ba65-4854a3ea-ff4d60a58aef. Acesso em: 03/set/2009.

NOSSA, Valcemiro; CHAGAS, José Ferreira. (1998). Usando programação linear na contabilidade decisorial. Revista Costos y Gestion. Buenos Aires, n. 27, mar. 
Utilização da Programação Linear na Otimização de Resultados de uma Empresa do Ramo de Transporte Rodoviário de Cargas Roberto Carlos Klann, Paulo Roberto da Cunha, Júlio Orestes da Silva, Jorge Eduardo Scarpin

OENNING, Vilmar et al. (2004). Teoria das restrições e programação linear. Uma análise sobre o enfoque de otimização da produção. In: ENCONTRO NACIONAL DE ENGENHARIA DE PRODUÇÃO - ENEGEP. 24, Florianópolis. Disponível em http://www.abepro.org.br/biblioteca/ENEGEP2004_Enegep0101_1441.pdf. Acesso em: 03/set/2009.

OJIMA, Andréa L. R. de Oliveira. (2007). Custo rodoviário como ferramenta de gerenciamento logístico para o transporte de soja: o caso da rota Barretos-Santos. Revista Informações Econômicas, São Paulo, v. 37, n. 5, p. 64-69, mai.

RAUPP, Fabiano Maury; BEUREN, Ilse Maria. (2006). Metodologia da pesquisa aplicável às ciências sociais. In: BEUREN, Ilse Maria (org.). Como elaborar trabalhos monográficos em contabilidade. (3 ed.). São Paulo: Atlas.

SEIXAS, Fernando; WIDMER, João A. (1993). Seleção e dimensionamento da frota de veículos rodoviários para o transporte principal de madeira utilizando-se de programação linear não inteira. IPEF, 46, p. 107-118, jan./dez.

SOTT, Valmir Roque. (2007). Lucro incremental e programação linear na tomada de decisões em ambiente de produção conjunta. Revista Gestão e Desenvolvimento em Contexto-Gedecon, v. 1, p. 108-136.

Data de Submissão: 13/12/2009

Data de Aceite: 23/03/2010 